\title{
EDUCATIONAL PEARL \\ Escape from Zurg: An Exercise in Logic Programming
}

\author{
MARTIN ERWIG \\ School of EECS, Oregon State University, Corvallis, Oregon 97331, USA \\ (e-mail: erwig@cs.orst.edu)
}

\begin{abstract}
In this article we will illustrate with an example that modern functional programming languages like Haskell can be used effectively for programming search problems, in contrast to the widespread belief that Prolog is much better suited for tasks like these.
\end{abstract}

\section{Introduction}

It is a common belief that Prolog is the language of choice to solve search problems. One strong point of Prolog is its built-in backtracking ability, which can save considerable work in handling search problems. On the other hand, Haskell (Peyton Jones, 2003) provides a powerful type system, higher-order functions, and lazy evaluation. We want to illustrate in this paper that these elements taken together make it as easy in Haskell (and maybe even easier) to express and solve search problems as it is in Prolog. For example, lazy evaluation facilitates the concise description of the search space because the specification of an infinite data structure - the search tree-can be written down without running into nontermination as long as only a finite part of it is processed. This idea is not new; it has been described by Phil Wadler (Wadler, 1985) before. However, rewriting this encoding for every search problem from scratch is tedious, error-prone, and can distract from the very search problem that is to be implemented. In Haskell, the concept of type classes offers a clean way to formulate the solution once and reuse it in different instances. Additionally, Haskell data types allow (and enforce to some degree) formulation of the problem in an adequate fashion.

An alternative approach is to embed a Prolog-like language into a functional language. This has been demonstrated in (Seres \& Spivey, 1999; Claessen \& Ljunglö, 2000) for Haskell and in (Haynes, 1987) for Scheme. However, our goal is to express search problems functionally without resorting to a multi-paradigm approach.

The example that we want to consider is a homework problem that we have given in a graduate level course on programming languages (Erwig, Fall 2001). The problem was one of several exercises to practice programming in Prolog. After observing that many students had problems manipulating term structures in Prolog (after already having learned to use data types in Haskell) and spending a lot of time on debugging, the question arose whether it would be as difficult to develop a solution for this problem in Haskell. This programming exercise worked well, and we report the result in this paper.

In the rest of this paper we will describe the requirements for teaching the programming 
of search problems in Haskell in Section 2. The example problem is described in Section 3. In Section 4 we show an example solution in Prolog. Section 5 presents the Haskell solution to the problem. Conclusion given in Section 6 complete this paper.

\section{Teaching Search Programming in Haskell}

To teach search programming in Haskell as proposed here, students should already have a solid understanding of essential functional programming concepts, such as, recursion, lists, and higher-order functions. In addition, students have to understand type classes, data types, and lazy evaluation, because these are used to create the modular solution.

First, a type class is used to separate the generic description of search problems from a particular problem instance. In particular, we make use of multi-parameter type classes to parameterize a search problem by the type of states and the type of moves. Multi-parameter type classes need not necessarily be known in advance. In fact, the SearchProblem class can serve as a motivating example to introduce multi-parameter type classes. The class itself can be developed in steps. Initially, a single-parameter version can be defined that is parameterized only over the type of search states. Then, recognizing that for some search problems, like the one discussed here, the solution states are not as interesting as the moves that lead to them, a generalization to two type parameters can be performed.

Second, data types are employed in the chosen example to create a model of the application. Data types provide a higher-level means of modeling the application than encoding all the information pieces by plain lists and tuples. Again, the encapsulation of the general search process in a type class is helpful since it allows us to focus completely on modeling the application because we do not have to deal with the search. This situation is similar in Prolog where the search procedure is built into the language. However, compared to Prolog terms, Haskell data types provide as a typed representation immediate feedback about illegal combinations of moves and states, which otherwise can cause a lot of debugging effort in untyped respresentations.

Third, knowledge of lazy evaluation is required to understand how the potentially infinite state space can be described in Haskell. A simple breadth-first search is implemented by creating a list of states through repeated appending of successor states. Depending on the focus and available time for dealing with search programming one might want to discuss this aspect in more depth. For example, it is relatively easy to generalize the class by parameterizing the construction of the search space by a search strategy. Again, since the search problem is isolated in a type class, this discussion does not affect the modeling of applications.

The implementation of search problems is discussed only in a few ML or Haskell textbooks. For example, Paulson (Paulson, 1996) describes the implementation of search programming in ML in the context of a theorem prover. Rabhi and Lapalme describe how to implement backtracking algorithms in Haskell (Rabhi \& Lapalme, 1999). They employ an explicitly defined depth-first search algorithm and do not use type classes to separate the problem class from the applications. In particular, they do not distinguish a separate type of moves, which makes the described approach inappropriate for the example problem discussed here. Felleisen et al. (Felleisen et al., 2001) describe in their book a similar example, the problem of three missionaries and cannibals crossing a river. This example 
is given as an exercise in the context of programming with generative recursion and accumulators, which are discussed in great depth as a means to retain context information in recursive function definitions.

\section{The Example Problem}

The problem to be solved was called "Escape from Zurg" and reads as follows:

Buzz, Woody, Rex, and Hamm have to escape from Zurg. ${ }^{a}$ They merely have to cross one last bridge before they are free. However, the bridge is fragile and can hold at most two of them at the same time. Moreover, to cross the bridge a flashlight is needed to avoid traps and broken parts. The problem is that our friends have only one flashlight with one battery that lasts for only 60 minutes (this is not a typo: sixty). The toys need different times to cross the bridge (in either direction):

$\begin{array}{lc}\text { ToY } & \text { TIME } \\ \text { Buzz } & 5 \text { minutes } \\ \text { Woody } & 10 \text { minutes } \\ \text { Rex } & 20 \text { minutes } \\ \text { Hamm } & 25 \text { minutes }\end{array}$

Since there can be only two toys on the bridge at the same time, they cannot cross the bridge all at once. Since they need the flashlight to cross the bridge, whenever two have crossed the bridge, somebody has to go back and bring the flashlight to those toys on the other side that still have to cross the bridge.

The problem now is: In which order can the four toys cross the bridge in time (that is, in 60 minutes) to be saved from Zurg?

${ }^{a}$ These are characters from the animation movie "Toy Story 2".

Try to solve the problem in your favorite language.

\section{A Prolog Solution}

Writing a Prolog program for solving the riddle is in principle a rather straightforward task-at least once it has been figured out how to represent the problem. As it turned out, this last aspect seemed to have been the major reason that quite a few students had problems with the assignment. The most difficult part for the students was to find an appropriate term representation for the states of the search problem, that is, the position of toys on either side of the bridge and the position of the flashlight. In particular, the two prevailing mistakes were to use too complex term structures or predicates and to use terms inconsistently, or even in a few cases to confuse predicates and terms. Several programs did not terminate. The reference solution is shown in Figure 1 to serve as a comparison with the Haskell solution to be developed in the next section.

The idea of the Prolog program is to represent an intermediate state of a bridge crossing 


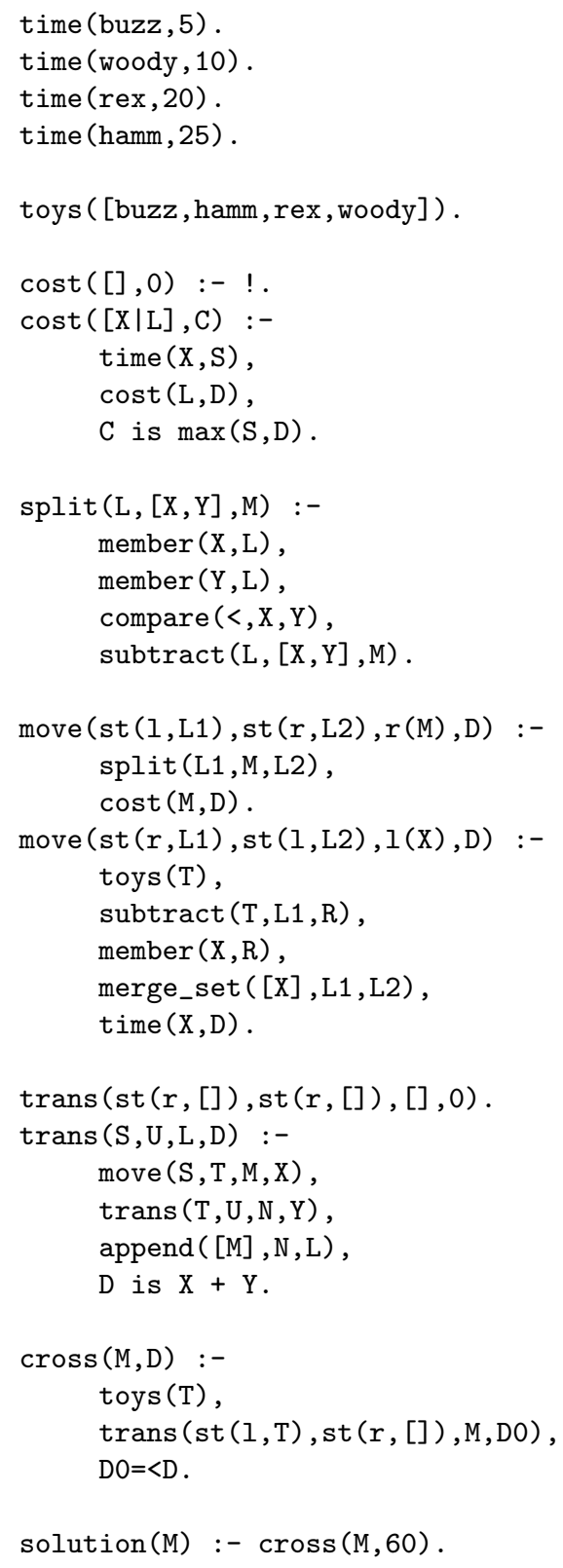

Fig. 1. Prolog solution for the Zurg riddle.

by facts of the form st $(P, L)$ where $L$ is a list giving the toys that are currently on the left side of the bridge and where $\mathrm{P}$ is a flag that indicates the position (left or right side) of the 
flashlight. ${ }^{1}$ The predicate move/4 generates movements in its third argument; a movement to the right is generated if the flashlight is on the left side of the bridge and vice versa; move also relates the old state (first argument) to the newly reached state (second argument). The last argument gives the time required for the move. In the case of a movement to the right, the time is determined by the additional predicate cost/ 2 that computes the maximum time needed by a group of toys. Any such possible group of toys to move to the right is computed by the predicate split/3, which computes lists of length 2 , which are sorted to avoid redundancy caused by representing groups of toys as lists. In a move to the left it makes only sense to send back one toy. Therefore, the definition of move for that case uses the predefined member $/ 2$ predicate and computes the time by simply looking into the table time/2. Finally, the trans/4 predicate basically generates all possible bridge crossings together with the required time whereas the cross $/ 2$ predicate formulates the search problem by giving the initial and final configuration of the search space.

\section{The Haskell Solution}

We give the Haskell solution in two steps. First, we extract the general structure of the search problem and capture it in the definition of a type class. Second, we present the program for solving the puzzle as an instance of that class.

The main elements in the problem are a state (to represent intermediate stages of bridge crossings) and moves (to represent transitions between states, which are in this case bridge crossings). Therefore, we have defined a type class SearchProblem with two parameter types $\mathrm{s}$ and $\mathrm{m}$. Next, we consider what member functions are needed for the class SearchProblem.

To build the complete search space starting from some (initial) state $\mathbf{s}$, a function is needed that describes which new states can be reached from s. In general, it is not only the final state that is of interest (in fact, we know this state already in the given example, namely all toys on the other side). Rather, the sequence of moves that lead to this state is needed, too. Therefore, we haved added a function to the type class that computes for a state a list of possible moves and the new states to which they lead:

trans : : $s \rightarrow[(m, s)]$

By repeatedly applying trans to the fringe of a search tree the complete search space for a problem can be constructed. This search space is represented by an element of type Space $\mathrm{m} \mathrm{s}$ and is constructed by a function space that maps a state to a list of all nodes of the search space. Each node (that is, state) is paired with the list of moves that lead to it.

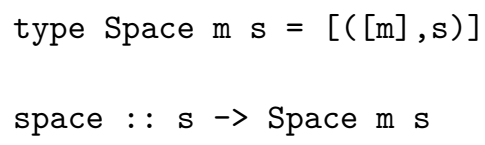

${ }^{1}$ In fact, more common among the students' solutions was the approach to represent two groups of toys on both sides of the bridge, but we found that although this redundancy might help to think about the problem, keeping the invariant was a common source of errors. 


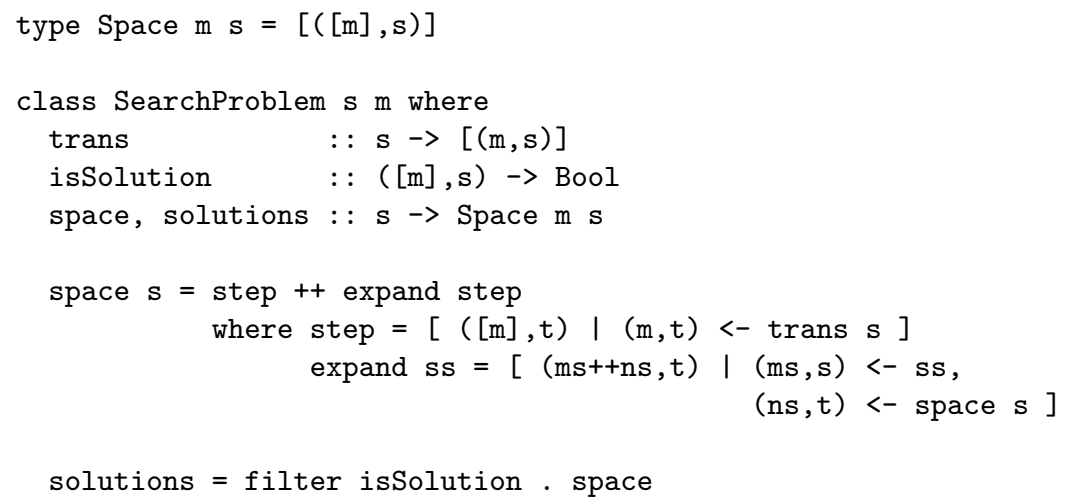

Fig. 2. The SearchProblem type class.

Since the search space is completely represented by the initial state and the function trans, the function space is a derived member function in the class SearchProblem. The definition of space makes essential use of lazy evaluation: without a terminating condition space refers (indirectly through expand) to itself; under strict evaluation this definition would, in general, not terminate. For the present example this means that it is enough to consider just two cases in the definition of trans (see Figure 3); an additional definition

$\operatorname{trans}(R,[])=[]$

is not needed, although such a condition was required in the Prolog program (cf. the first clause for the predicate trans).

In general, the solutions to a search problem are given by a subset of its states. The decision is made by a predicate on states and their generating moves:

isSolution : : $([\mathrm{m}], \mathrm{s}) \rightarrow$ Bool

With this predicate another class member solutions can be defined, which has the same type as space and which simply yields the subset of states that are considered solutions by the predicate isSolution. The definition of the type class SearchProblem is summarized in Figure 2.

Having defined the solution schema, the Haskell program for solving the riddle requires only the modeling of the problem. The most important design decisions are the definitions of the types BridgePos and Move because they are related by an instance definition for the class SearchProblem. In BridgePos we represent the position of the flashlight by a constructor $\mathrm{L}$ or $\mathrm{R}$ and the toys that are on the left side of the bridge by a list, just like in the Prolog implementation. A move is either a move of a group of toys from left to right or a backward move from right to left by just one toy. Both kinds of moves are captured by the type Move, which is defined through an Either data type, which is predefined in Haskell and which contains the constructors Left and Right to represent disjoint sum types.

Apart from defining types for representing the objects in the program, the main part is the instance definition of the SearchProblem type class, which means to give a definition for 


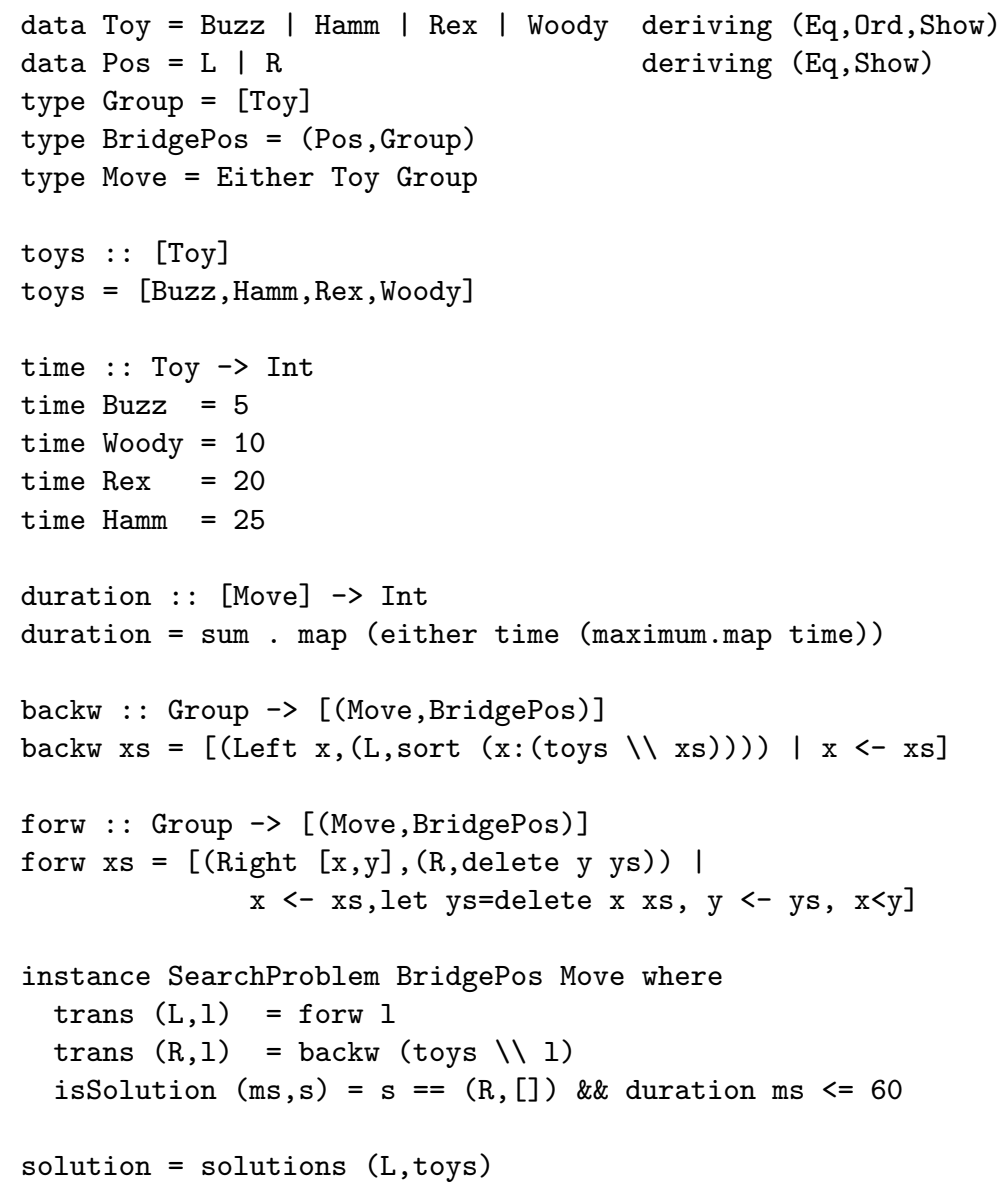

Fig. 3. Haskell solution for the Zurg riddle.

trans and isSolution. To this end, we have defined three auxiliary functions: forw and backw for computing toy moves and duration for computing the total time of a crossing, that is, for a sequence of moves. Note that the function $(\backslash \backslash)$ computes the difference of two lists. The definition of the isSolution predicate is obvious. The complete Haskell solution is shown in Figure 3.

We have already mentioned that the SearchProblem type class from Figure 2 implements a simple breadth-first search. A generalization can be obtained by abstracting from the append operation that is used to add newly generated states to the list of states, that is, we introduce a function parameter into the definition of space and solutions that controls the addition of new states to the space. A possible implementation is shown in Figure 4.

To use this generalized type class for the example problem, we only have to pass a corresponding search strategy to the solutions function, for instance: 


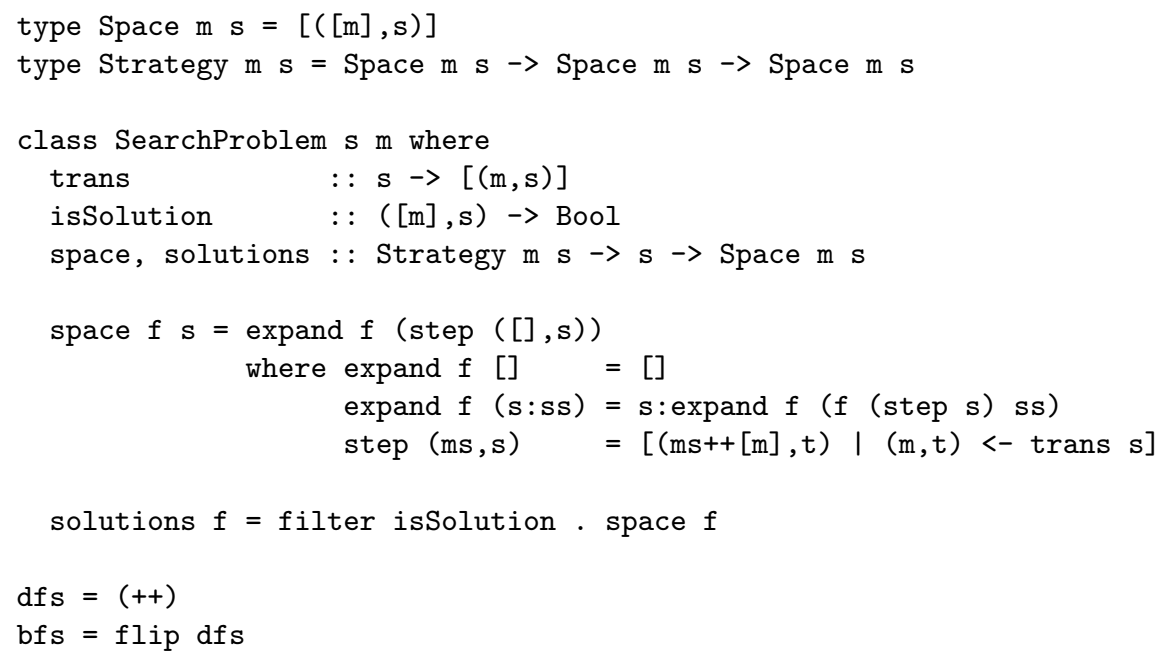

Fig. 4. Generalized SearchProblem type class.

solution = solutions bfs (L,toys)

For the example problem, the search strategy does not affect the solution, but for other search problems, termination is generally more likely under bf $\mathrm{s}$ than under $\mathrm{df} \mathrm{s}$.

\section{Conclusions}

Let us first summarize our experience with the shown programming exercise. Most students seemed to like the puzzle style of the assignment, although quite a few students had problems during the development of their solution and had to spend a considerable amount of time debugging their programs. A particular problem was to spot illegal uses of Pro$\log$ terms that showed up in the interpreter just through the answer No. Another mistake was to confuse terms and predicates. To some degree, the completely different lexical conventions in Haskell and Prolog are probably responsible for this confusion: Variables start with an uppercase letter in Prolog, and with a lowercase letter in Haskell, whereas term constructors start with a with a lowercase letter in Prolog, and with an uppercase letter in Haskell.

Some students tried to work around their problems by coding knowledge about the solution into their programs, for example, fixing the number of forward and backward moves in the problem representation. Some of the solutions handed in by students were similar to the one shown in Figure 1, differing mainly in the chosen term representation and in how the transition predicate was defined. An incorrect term representation was the main problem for those program that did not run at all or that were computing incorrect results.

To obtain feedback about the Haskell approach, a couple of graduate students were asked to solve the problem also in Haskell. All they were given was the definition of the SearchProblem type class. Those students who already got the Prolog solution correct 
reported that it was as easy in Haskell to come up with a solution as in Prolog. Others who had non-perfect Prolog solutions felt it was easier to write the Haskell program than the Prolog program. They also reported that the type system was helpful in designing the solution and in debugging the program.

From our experience with solving the example problem with both languages, we believe that Haskell's type system makes it eventually easier to implement search problems in Haskell than in Prolog. The most important feature of Haskell that supports this impression is the availability of multi-parameter type classes, because we can abstract the general solution schema in a type class and reuse it for other problems.

\section{Acknowledgments}

The author thanks Matthias Felleisen for his valuable hints and remarks that helped to improve this paper. Many thanks also go to the students of the programming languages class who provided a lot of feedback about their experience with Haskell, Prolog, types, etc.

\section{References}

Claessen, K., \& Ljunglö, P. (2000). Typed Logical Variables in Haskell. Haskell Workshop. Electronic Notes in Theoretical Computer Science, Vol. 41, No. 1.

Erwig, M. (Fall 2001). CS 581: Programming Languages. Graduate Course. Department of Computer Science, Oregon State University. http://www.cs.orst.edu/ ${ }^{\sim}$ erwig/old/cs581.f01.

Felleisen, M., Findler, R. B., Flatt, M., \& Krishnamurthi, S. (2001). How to Design Programs-An Introduction to Programming and Computing. Cambridge, MA: MIT Press.

Haynes, C. T. (1987). Logic Continuations. The Journal of Logic Programming, 4, 157-176.

Paulson, L. C. (1996). ML for the Working Programmer (2nd ed.). Cambridge, NY: Cambridge University Press.

Peyton Jones, S. (2003). Haskell 98 Language and Libraries. Cambridge, UK: Cambridge University Press.

Rabhi, F., \& Lapalme, G. (1999). Algorithms: A Functional Programming Approach. Harlow, England: Addison-Wesley.

Seres, S., \& Spivey, M. (1999). Embedding Prolog in Haskell. Haskell Workshop. Technical Report UU-CS-1999-28, Universiteit Utrecht.

Wadler, P. (1985). How to Replace Failure by a List of Successes. Pages 113-128 of: Conf. on Functional Programming and Computer Architecture. LNCS 201. 